\title{
Implementation of a Fused Approach for Segmentation of Brain MR Images for Tumor Extraction
}

\author{
Shimpa Sethi \\ Shaheed Bhagat Singh State Technical Campus \\ Ferozepur, Punjab, India
}

\author{
Jaswinder Kaur \\ Shaheed Bhagat Singh State Technical Campus, \\ Ferozepur, Punjab, India
}

\begin{abstract}
Medical image segmentation plays an important role in diagnosis and various medical evaluations. Detection and segmentation of Brain tumor accurately is a challenging task. Different kinds of segmentation algorithms have been proposed for image segmentation. In this paper, a method is proposed that integrates advanced K-Means clustering and marker controlled watershed segmentation algorithm for MRI images of brain. The Enhanced K-means clustering is used to produce a primary segmentation of the image before applying marker controlled watershed segmentation algorithm to it. It has been shown that proposed method is able to eliminate over segmentation problem which generally occurs in case of conservative watershed algorithm.
\end{abstract}

\section{Keywords}

Medical Imaging, Brain Tumor, MRI, K-means Clustering, Marker Controlled Watershed Segmentation.

\section{INTRODUCTION}

Image segmentation is the division of an image into different regions, each possessing particular properties. In a segmented image, the elementary picture elements are the connected sets of pixels belonging to the same region. Once the image has been segmented, the processing can be done on each of the regions. Image segmentation is therefore a key step towards the quantitative interpretation of image data [1].

Segmentation of intensity images usually involves five main approaches, namely threshold, boundary detection, region-based processing, pixel intensity and morphological methods. In this paper, we have integrated one of the region based approach known as clustering and a well known morphological approach to segmentation, known as watershed algorithm [2]. This paper describes the detection of the brain tumor by segmentation using proposed technique. The processing of magnetic resonance images (MRI) is one among the parts of the image processing in medical field. MRI is an essential process for detection and diagnosis of any brain abnormalities or lesions. This process is performed visually by trained radiologists with a significant degree of precision and accuracy. Accurate segmentation is still a challenging task because of the variety of the possible shapes, locations and image intensities of various types of problems and protocols [3].

Computerized segmentation may help the radiologists to segment the region of interest precisely. Thus image segmentation becomes more significant while normally dealing with medical images. The proposed technique can be efficiently applied to detect and extract the brain tumor from MRI image [4].
A brain tumor is an abnormal growth of cells within the brain, which can be cancerous or non-cancerous (benign).It is defined as any intracranial tumor created by abnormal and uncontrolled cell division, normally either in the brain itself (neurons, glial cells (astrocytes, oligodendrocytes, ependymal cells), lymphatic blood vessels), in the cranial nerves (myelin-producing Schwann cells), in the brain envelopes (meninges), skull, pituitary and pineal gland, or spread from cancers primarily located in other organs (metastatic tumors) [5]. A brain tumor takes up space within the skull and can interfere with normal brain activity. It can increase pressure in the brain, shift the brain or push it against the skull, and/or invade and damage nerves and healthy brain tissue. The location of a brain tumor influences the type of symptoms that occur. Identifying the presence of a brain tumor is the first step in determining a course of treatment.

\section{BACKGROUND AND RELATED WORK}

Diagnostic imaging is an invaluable tool in medicine. Magnetic resonance imaging (MRI), computed tomography (CT), digital mammography, and other imaging modalities provide an effective means for non- invasively mapping the anatomy of a subject. These technologies have greatly increased knowledge of normal and diseased anatomy for medical research and are a critical component in diagnosis and treatment planning. The growing size and number of medical images have necessitated the use of computers to facilitate processing and analysis. In particular, computer aided algorithms for the demarcation of anatomical structures and other regions of interest are becoming increasingly important in assisting and automating specific radiological tasks. These algorithms, called image segmentation algorithms, play a vital role in numerous biomedical-imaging applications, such as the quantification of tissue volumes, diagnosis, localization of pathology, study of anatomical structure, treatment planning, and computer-integrated surgery. A variety of examinations are performed to diagnosis a brain tumor. Magnetic resonance imaging (MRI) and computed tomography (CT or CAT scan) are the most common types of scans used to diagnose brain tumors. Both MRI and CT scan use computers to create detailed images of the brain. The other methods include PET (Positron Emission Tomography) scan, DTI ( Diffusion Tensor Imaging), MRS (Magnetic Resonance Spectroscopy) scan and biopsy (tissue sample analysis) [6-7].

The computer-assisted diagnostic methods are robust and significantly faster. These methods assist the radiologists in fast diagnosis and further treatment planning. They are capable of producing better results as compared to traditional methods.

Anil z chitade et. al. [9] presented a novel image segmentation based on color features with k-means clustering unsupervised 
algorithm. S. Thilagamani et al. [10] presented a survey on different clustering techniques to achieve image segmentation. Clustering is done based on different attributes of an image such as size, color, texture etc. Kanungo et al. [12] proposed a simple and efficient implementation of Lloyd's algorithm, which we call the filtering algorithm before applying K means. Ahmad el allaoui et al. [16] proposed some improvements to watershed algorithm based on the mathematical morphology in order to get over difficulty of over segmentation. Amir Shahzad et al. [17] presented an algorithm which is based on merging watershed result with enhanced edge detection result. Petros Karvelis et al. [18] proposed a novel segmentation method that tackles the problem of touching-overlapping group of chromosomes.

So far, a number of methods have been developed with the aim to identify the distinct region of objects in the image. This work aims to make a combination of enhanced K-means clustering and improved watershed algorithm used for image segmentation to remove the over segmentation problem in the segmented images. This algorithm has better ability of segmentation and solving over-segmentation problem [8].

Before using improved watershed segmentation method, the image was enhanced using morphological operations and then segmented primarily using K-means clustering method which becomes enhanced K-means clustering algorithm.

\section{PROPOSED METHODOLOGY}

The computations for proposed method are compiled using MATLAB Image Processing Toolbox. The proposed methodology is a two stage process. The first process uses enhanced K-means clustering to produce a primary segmentation of the input image, while the second process applies the marker controlled watershed segmentation algorithm to the primary segmentation to produce the final result of segmentation.

\subsection{K-Means Clustering}

K-means clustering algorithm, which is an unsupervised method, the idea is to classify a given set of data into $\mathrm{k}$ number of disjoint clusters, to provide us with a primary segmentation of the image. It is observed that there are many regions with similar intensities in a MR image of the head, which result in many local minima that increases over segmentation, when we apply the watershed algorithm. The coarse areas are smoothened in the primary segmentation. K-means clustering is used because it is simple and has relatively low computational complexity. In addition, it is suitable for biomedical image segmentation as the number of clusters $(\mathrm{K})$ is usually known for images of particular regions of human anatomy. MR image of the head generally consists of regions representing the bone, soft tissue, fat and background. Further the K-means algorithm can be slightly modified to produce better results for primary segmentation [9-10].

\subsection{Enhanced K-Means Clustering}

Morphological reconstruction before applying the basic Kmeans algorithm gives better results than the direct application of basic k-means algorithm .Morphological reconstruction is used to enhance the clusters formed in the image. Morphological processing is based on the set theory to provide basic tools to deal with images for filtering, thinning and pruning operations that are useful for the description of region shape involving boundary and skeleton representation. Dilation and erosion are the two fundamental operations in morphological processing. Our Morphological processing algorithms are based on the specific combination of the dilation and erosion operations. We have used morphological techniques called "opening-by-reconstruction" and "opening -closing-byreconstruction" to clean up the image.

Opening is an erosion followed by dilation, while opening-byreconstruction is an erosion followed by a morphological reconstruction. Opening-by-reconstruction is computed using imerode and imreconstruct command in Matlab. Next closingby-reconstruction is computed using imdilate followed by imreconstruct command in MATLAB [11-13].

\subsection{Watershed Algorithm}

The concept of Watersheds can also be used for image segmentation. There are different algorithms to compute watersheds. Watershed algorithm is sensitive to weak edges. Although it overcame the shortcomings of traditional segmentation method for losing the weak edge, but it could also detect low contrast change in the region of symmetrical area, resulting in over segmentation problems. Therefore, in the application of watershed image segmentation algorithm, image preprocessing or post-processing are often made to limit the number of the regions and the gradient magnitude is obtained by applying the prewitt operator. Further the improved watershed method has been applied known as Marker Controlled Watershed Segmentation Algorithm [14].

\subsection{Marker Controlled Watershed Segmentation Algorithm.}

Segmentation using the watershed transforms works well if we can identify, or mark, foreground objects and background locations. Marker-controlled watershed segmentation follows this basic procedure:

1. Compute a segmentation function. This is an image whose dark regions are the objects we are

trying to segment.

2. Compute foreground markers. These are connected blobs of pixels within each of the objects.

3. Compute background markers. These are pixels that are not part of any object.

4. Modify the segmentation function so that it only has minima at the foreground and

background marker locations.

5. Compute the watershed transform of the modified segmentation function [15-16].

\section{RESULTS}

This section presents our results on the segmentation of brain tumor done with the help of proposed technique. The enhanced $\mathrm{K}$-means clustering algorithm is used to improve the accuracy of the segmentation from the target area. The use of enhanced K-means clustering before applying marker controlled watershed segmentation algorithm has achieved the objective of reducing the problem of over segmentation when applied to MR brain images. The foreground objects and background objects are marked by opening followed by closing by reconstruction. After this operation, limited number of region minimum is obtained which helps in separating the region of interest from rest of the image. The analysis of results show that the proposed method can effectively segment the tumor part of different sizes and shapes and is precise, simple and robust. The segmentation results for three Brain MR Scan images are shown in Fig.1, 2 and 3 . 

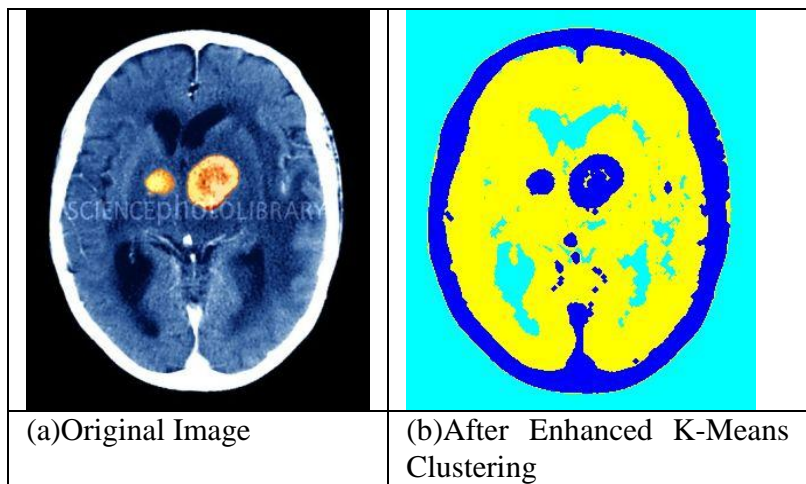

(a)Original Image

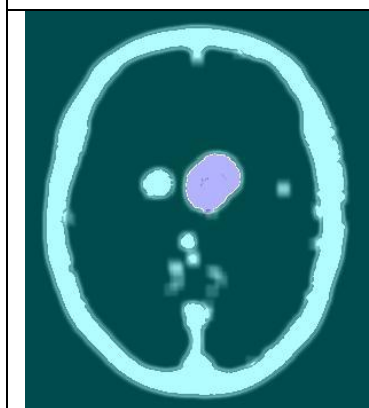

(c)Segmentation By Proposed Algorithm

Fig 1: Brain MR Scan 1

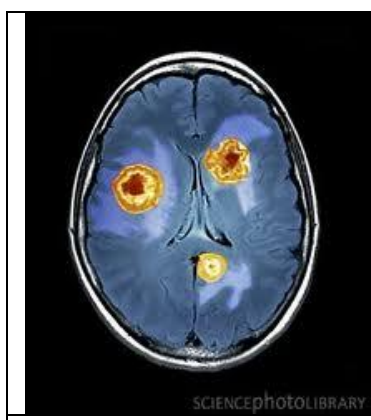

(a)Original Image

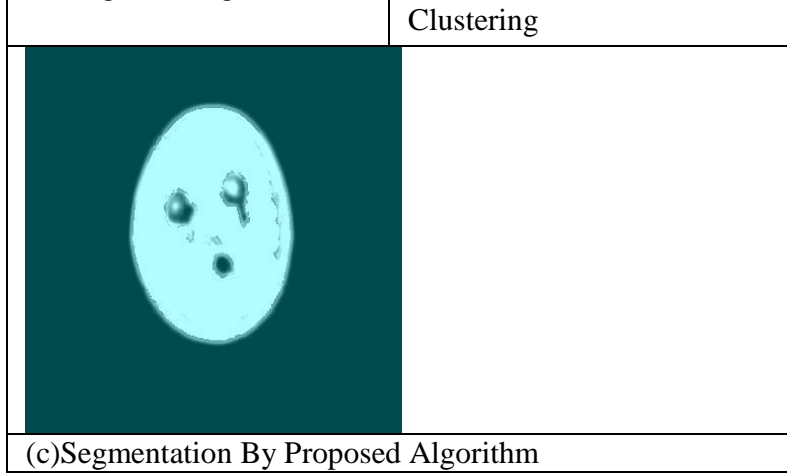

Fig 2: Brain MR Scan 2
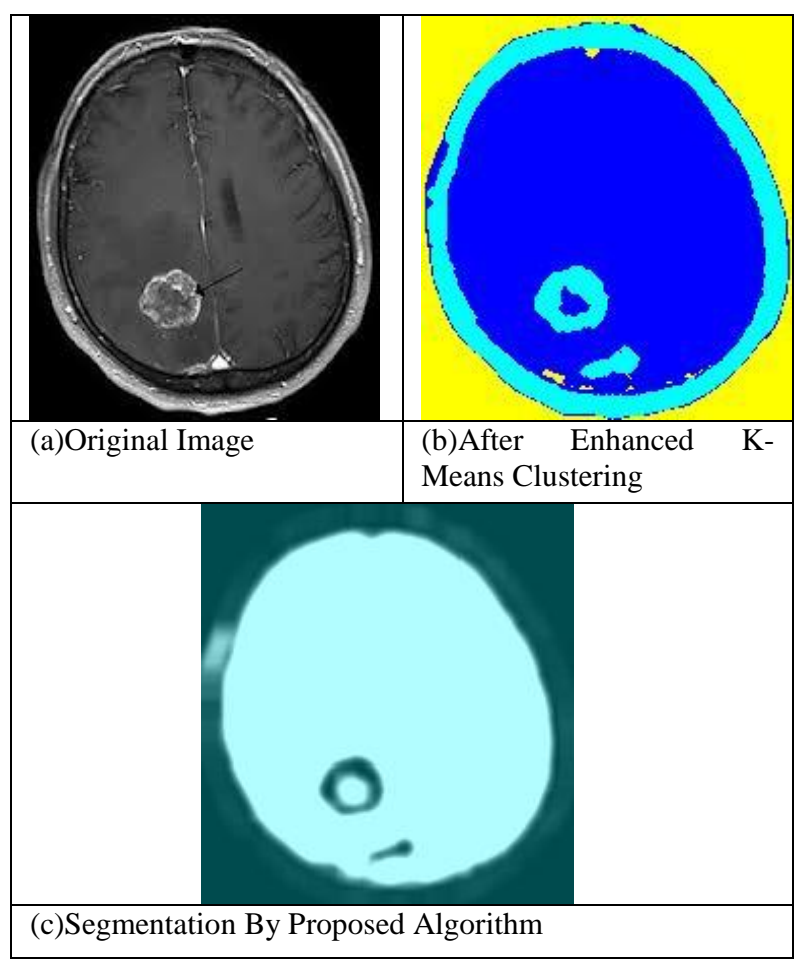

Fig 3: Brain MR Scan

\section{CONCLUSION}

K-Means Clustering and Watershed Transform image segmentation algorithms have been studied. It is therefore concluded that the direct Watershed implementation exhibits poor segmentation maps and segmentation is not successful. Finally the over-segmentation problem of Watershed is addressed by combining enhanced K-Means and Marker Controlled Watershed algorithms. The resulted algorithm inherits the performances of its parent algorithms and provides better results compared to the conventional $\mathrm{K}$ - means clustering algorithm and conventional watershed algorithm. It also has a certain degree of robustness and accuracy.

\section{FUTURE SCOPE}

Medical image computing has become one of the most challenging fields in medical informatics. Furthermore, in the field of software-assisted and navigated surgery medical image computing methods play a key role and have opened up new perspectives for patient treatment. These methods enable new insights into the patient's image data and have the future potential to improve medical diagnostics and patient treatment. We have referred to the most commonly used radiological modalities for imaging anatomy. Most of the concepts described could be valid for other imaging techniques as well. Also further developments are needed to increase the rank of computerization, precision and robustness.

\section{REFERENCES}

[1] Manjusha Singh, Abhishek Misal (2013): 'A Survey Paper on Various Visual Image Segmentation Techniques', International Journal of Computer Science and Management Research, Volume 2, issue 1, pp.1282-1288, ISSN 2278-733X. 
[2] Rajeshwar Dass, Priyanka, Swapna Devi (Jan-March 2012):'Image Segmentation Techniques', International Journal of Electronics \& Communication Technology, Volume 3, Issue 1, ISSN 2230-7109.

[3] Anjum Hayat Gondal Muhammad Naeem Ahmed Khan (2013): 'A Review of Fully Automated Techniques for Brain Tumor Detection from MR Images', I.J.Modern Education and Computer Science 2, pp. 55-61.

[4] Image Courtesy: Science Photo Library available at link:http://www.sciencephoto.com/image/158131/530wm/ C0095518Secondary_brain_cancer,_MRI_scan-SPL.jpg and Science and Life available at link: http://sciencemedicine-life.blogspot.in/

[5] Des Plaines, IL (1998): 'A Primer of Brain Tumors-a patients' reference manual', American Brain Tumor Association (1973).

[6] Preeti Aggarwal, Renu Vig, Sonali Bhadoria, C.G.Dethe (sep.2011): Role of Segmentation in Medical Imaging: A Comparative Study', International Journal of Computer Applications (0975 - 8887) Volume 29- No.1, pp. 54-61.

[7] Laura Drever, Wilson Roa, Alexander McEwan and Don Robinson(2007):Comparison of three image segmentation techniques for target volume delineation in positron emission tomography, Journal Of Applied Clinical Medical Physics, Volume 8, Number 2.

[8] Tuhin Utsab Paul, Samir Kumar Bandhyopadhyay, (MayJun 2012): 'Segmentation of Brain Tumor from Brain MRI Images Reintroducing K - Means with advanced Dual Localization Method", International Journal of Engineering Research and Applications (IJERA), ISSN: 2248-9622 Vol. 2, Issue 3, pp. 226-231 226.

[9] Anil z chitade et. Al. (2010): 'Colour based image segmentation using k-means clustering', International journal of engineering science and technology vol. 2(10), pp.5319-5325.

[10] S. Thilagamani and N. Shanthi (March 2011):"A Survey on Image Segmentation Through Clustering, International
Journal of Research and Reviews in Information Sciences Vol. 1, No. 1.

[11] K. Parvati, B. S. Prakasa Rao, and M. Mariya Das (2008): 'Image Segmentation Using Gray Scale Morphology and Marker-Controlled Watershed Transformation', Hindawi Publishing Corporation Discrete Dynamics in Nature and Society Volume 2008, Article ID 384346, 8 pages.

[12] Tapas Kanungo, David M Mount (July 2002): 'An Efficient K-means Clustering Algorithm: Analysis and Implementation', Pattern Analysis and Machine Intelligence, IEEE Transactions on Pattern Analysis and Machine Intelligence. Vol. 24, No. 7.

[13] Rafael C. Gonzalez, Richard E. Woods., Steven L. Eddins (2004): Digital Image Processing Using MATLAB, Pearson Education Inc, 2004

[14] M.C. Jobin Christ and R.M.S. Parvathi (2011): 'Segmentation of Medical Image using Clustering and Watershed Algorithm', American Journal of Applied Sciences 8 (12): pp.1349-1352, 2011 ISSN 1546-9239.

[15] M. C. Jobin Christ , R. M. S. Parvathi(2012): 'Segmentation of Medical Image using KMeans Clustering and Marker Controlled Watershed Algorithm', European Journal of Scientific Research ISSN 1450-216X Vol.71 No.2, pp. 190-194.

[16] Ahmad El Allaoui and M'barek Nasri (June 2012): 'Medical Image Segmentation by Marker-Controlled Watershed and Mathematical Morphology', International Journal of Multimedia \& Its Applications (IJMA) Vol.4, No.3, pp. 1-9.

[17] Amir Shahzad, Muhammad Sharif, Mudassar Raza, Khalid Hussain (2008): 'Enhanced Watershed Image Processing Segmentation', Journal of Information \& Communication Technology Vol. 2, No. 1, (Spring 2008) 01-09.

[18] Petros Karvelis, Aristidis Likas , Dimitrios I. Fotiadis(2010): 'Identifying touching and overlapping chromosomes using the watershed transform and gradient paths', Pattern Recognition Letters 31, pp.2474-2488. 